\title{
Plasmon-Enhanced Sensing: Current Status and Prospects
}

\author{
Jiangtao Lv, ${ }^{1}$ Eunice Sok Ping Leong, ${ }^{2}$ Xiaoxiao Jiang, ${ }^{1}$ Shanshan Kou, ${ }^{3,4}$ \\ Haitao Dai, ${ }^{5}$ Jiao Lin, ${ }^{6,7}$ Yan Jun Liu, ${ }^{2}$ and Guangyuan $\mathrm{Si}^{1,6}$ \\ ${ }^{1}$ College of Information Science and Engineering, Northeastern University, Shenyang 110004, China \\ ${ }^{2}$ Institute of Materials Research and Engineering, Agency for Science, Technology and Research (A*STAR), 3 Research Link, \\ Singapore 117602 \\ ${ }^{3}$ Australian Research Council Centre of Excellence (ARC CoE) for Advanced Molecular Imaging, La Trobe University, Melbourne, \\ VIC 3086, Australia \\ ${ }^{4}$ Department of Chemistry and Physics, La Trobe Institute for Molecular Sciences (LIMS), La Trobe University, Melbourne, \\ VIC 3086, Australia \\ ${ }^{5}$ Tianjin Key Laboratory of Low Dimensional Materials Physics and Preparing Technology, School of Science, \\ Tianjin University, Tianjin 300072, China \\ ${ }^{6}$ School of Electrical and Computer Engineering, RMIT University, Melbourne, VIC 3001, Australia \\ ${ }^{7}$ School of Physics, The University of Melbourne, Melbourne, VIC 3010, Australia
}

Correspondence should be addressed to Yan Jun Liu; liuy@imre.a-star.edu.sg and Guangyuan Si; siguang0323@hotmail.com

Received 10 September 2015; Accepted 5 November 2015

Academic Editor: Kimberly Hamad-Schifferli

Copyright (C) 2015 Jiangtao Lv et al. This is an open access article distributed under the Creative Commons Attribution License, which permits unrestricted use, distribution, and reproduction in any medium, provided the original work is properly cited.

By combining different plasmonic nanostructures with conventional sensing configurations, chemical/biosensors with significantly enhanced device performance can be achieved. The fast development of plasmon-assisted devices benefits from the advance of nanofabrication technology. In this review, we first briefly show the experimental configurations for testing plasmon enhanced sensing signals and then summarize the classic nanogeometries which are extensively used in sensing applications. By design, dramatic increment of optical signals can be obtained and further applied to gas, refractive index and liquid sensing.

\section{Introduction}

The rapid development of plasmonics [1-3] and plasmonrelated devices [4-12] paves the way for controlling electromagnetic waves at the nanoscale. The coherent free electron excitations (i.e., surface plasmon resonances, SPRs) which exist at the metal/dielectric interfaces are normally generated by illuminating light to metallic or metallic-dielectric hybrid structures. As yet, various plasmon-assisted optical components have been conceived and experimentally demonstrated, including waveguides [13-19], photon sorters [2022], absorbers [23-25], color filters [26-28], and switches $[29,30]$. SPR based optical sensors [31-56] are another important research field since they have found numerous useful applications in detecting and characterizing chemical and biological molecules.
It is well known that surface plasmons can propagate along the metal-dielectric interface from tens to hundreds of microns and decay evanescently in the vertical direction. Based on the fact that SPR is sensitive and highly dependent on the dielectric environment, the shift of resonance in optical spectrum can be used to quantify the change of surrounding medium since analyte can interact with electromagnetic waves which are tightly confined on the structure surface. Note that nonpropagating plasmons are also useful for sensing applications due to greatly enhanced field intensity at the resonance (localized surface plasmon resonance, LSPR) due to collective oscillations. Here, we first briefly retrospect the classical experimental configurations for plasmon-enhanced sensing and then summarize typical designs that can remarkably strengthen the optical signal. 


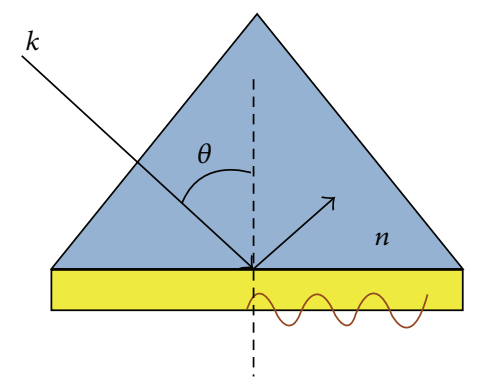

(a)

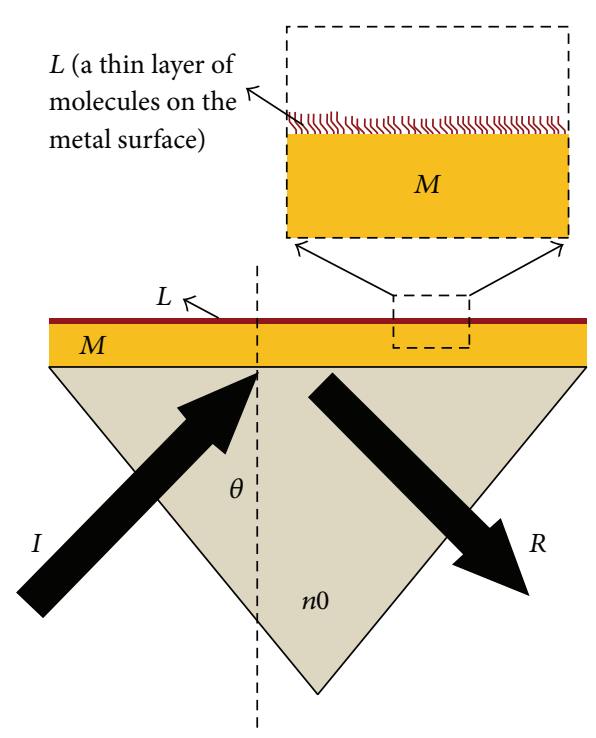

(c)

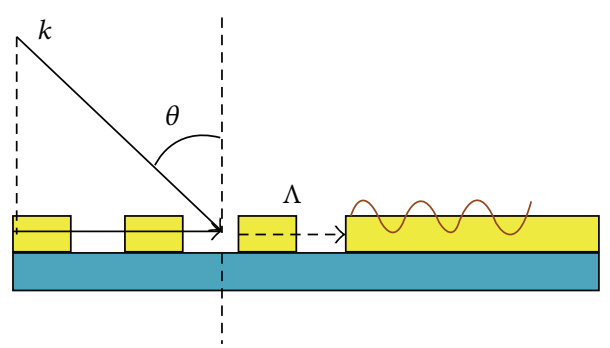

(b)

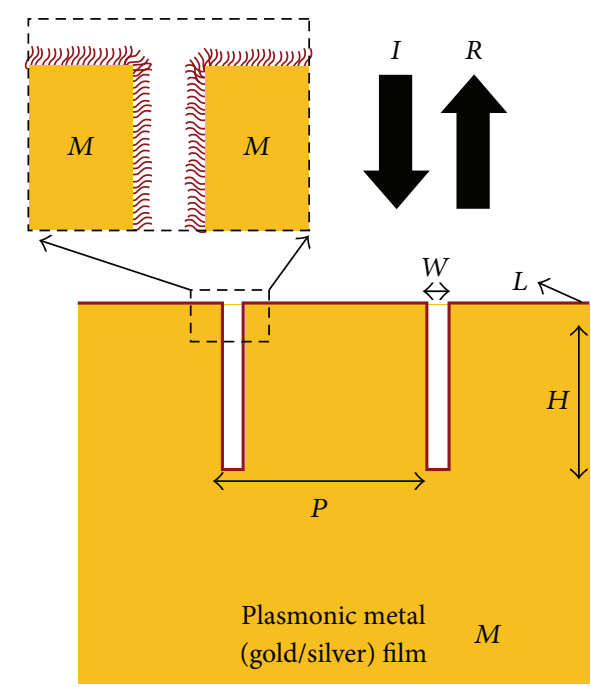

(d)

FIGURE 1: (a) Schematic showing the Kretschmann configuration for surface plasmon excitation. (b) Grating can also provide additional wave vector components and therefore assist the conversion from incident light into surface plasmon waves. (c) Schematic diagram showing Kretschmann configuration conventionally employed for coupling incident radiation to surface plasmons with a thin layer of molecules on the metal surface. (d) Narrow groove plasmonic grating structures illustrating the important dimensions and parameters. The incident and reflected radiation are indicated by symbols " $I$ " and " $R$," respectively. (a)-(b) and (c)-(d) are adapted from [57, 58], respectively. (a)-(b): Copyright 2014, Multidisciplinary Digital Publishing Institute. (c)-(d): Copyright 2011, Optical Society of America.

The sensing effect will be reviewed both theoretically and experimentally.

\section{Configurations of SPR-Assisted Sensing}

Figure 1(a) illustrates the classic Kretschmann configuration [57] which is most frequently applied to excite SPR. A prism is normally used to match the wave vectors between surface plasmons and the incident light. Alternatively, patterned structures (e.g., gratings) can also be employed to generate surface plasmons, as shown in Figure 1(b). Additional wave vectors are offered by patterned structures which further help convert the incident light to surface plasmon waves.

To take advantage of the unique properties of SPR, one needs to combine the analyte with experimental setup which can excite surface plasmons. This can be realized by covering the metal surface with a thin layer of molecules [58], as illustrated in Figure 1(c). More complicatedly, complex designs of nanostructures with well-aligned arrays can be utilized to further generate optical response at different frequency bands. Analyte tightly adhered to patterns (e.g., nanotrenches) as shown in Figure 1(d) interacts with excited SPR and produces detectable resonance shift in the spectrum.

\section{Plasmonic Nanostructures for Enhanced Sensing Applications}

A variety of patterning methods have been developed to fabricate nanostructures which can be further used for enhancing the serviceable signals. Typically, two different processes are categorized: top-down and bottom-up fabrication techniques. The former includes focused ion beam (FIB) milling [59-62], electron-beam lithography (EBL) [63-67], nanoimprint [68-71], and interference lithography [72-75]. Various plasmonic designs have been demonstrated to enhance the sensing effect. Figure 2 shows typical structures including 1D nanogratings (Figure 2(a)), 2D nanodots (Figure 2(b)), nanoholes (Figure 2(c)), and nanomushrooms (Figure 2(d)). 


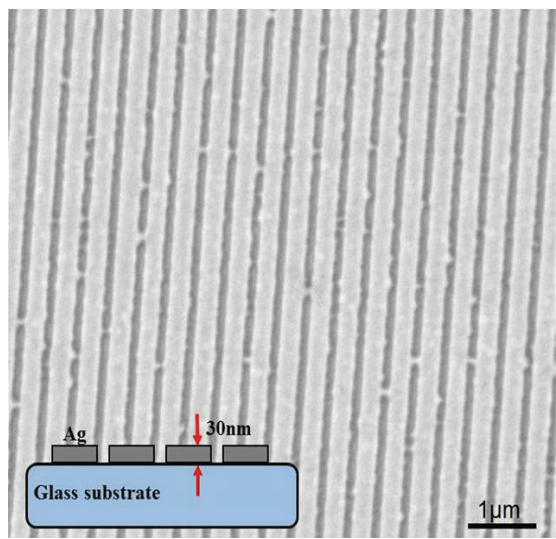

(a)

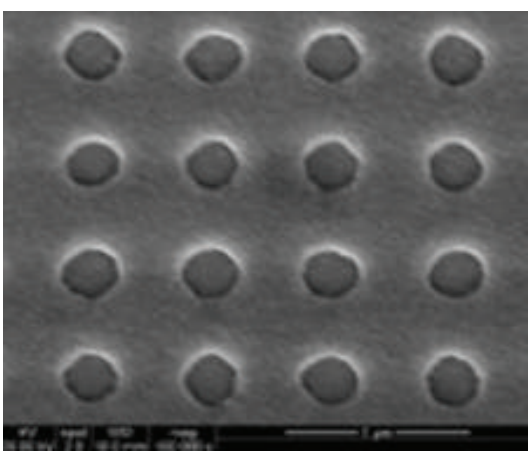

(c)

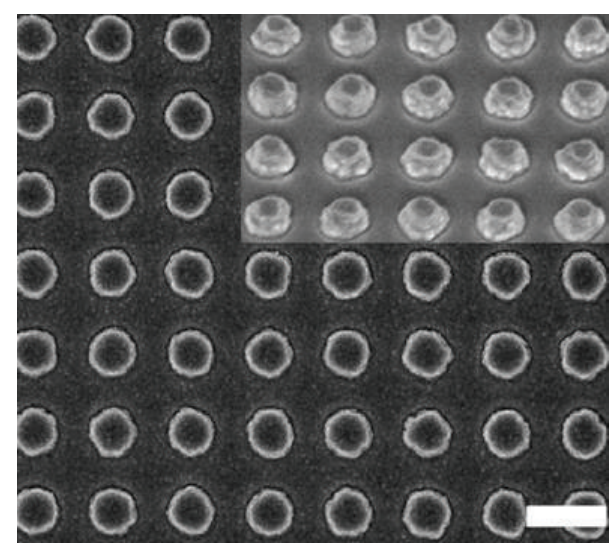

(b)

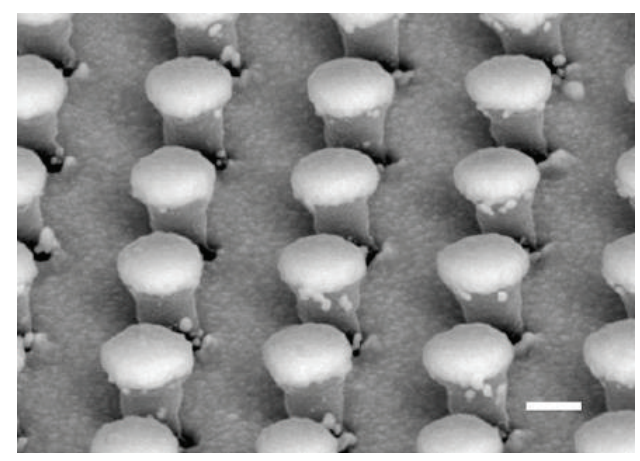

(d)

FIGURE 2: SEM images showing four different plasmonic nanodesigns. (a) SEM image of 1D nanogratings patterned on a silver film with $420 \mathrm{~nm}$ period and $110 \mathrm{~nm}$ slit-width. The inset illustrates the schematic cross section of the nanogratings on a glass substrate. (b) Top-view SEM image of a nanodisk array with $500 \mathrm{~nm}$ periodicity. The inset is the oblique view of the disks. (c) Top-view SEM image of a nanohole array. (d) Oblique view of nanomushrooms fabricated by interference lithography and thermal evaporation. Scale bars in (b) and (d) represent $300 \mathrm{~nm}$ and $200 \mathrm{~nm}$, respectively. (a), (b), (c), (d) are successively adapted from [76-79]. (a): Copyright 2014, American Institute of Physics. (b): Copyright 2014, Elsevier. (c): Copyright 2014, American Institute of Physics. (d): Copyright 2013, Nature Publishing Group.

FIB milling was applied to fabricate the nanogratings illustrated in Figure 2(a) [76]. One can see that the sidewalls are not completely straight, which is caused by material redeposition during milling. Although the redeposition effect is almost inevitable in all ion-involved milling processes, it is still possible to minimize the effect and obtain a smooth surface under optimized conditions. Large area 2D nanorods [77] and nanoholes [78] shown in Figures 2(b) and 2(c) were fabricated by interference lithography. Note that either lift-off or etching is needed to transfer patterns from resists to target materials using interference lithography to define patterns, which is different from direct FIB drilling. It is also worth mentioning that the cross sections of the fabricated structures may not present vertical sidewalls, as illustrated in the inset of Figure 2(b). Using interference lithography followed by thermal evaporation, nanomushrooms (gold caps on photoresist pillars) [79] shown in Figure 2(d) were obtained on a quartz substrate. One can also observe fabrication imperfections from the SEM image.

Except for the regular structures (well-aligned arrays) discussed above, various designs with irregular shapes have also been reported. As shown in Figures 3(a) and 3(b), gold nanoislands [80, 81] were constructed by evaporation followed by annealing, which can be used for chemical/ biosensing in the transmission localized surface plasmon resonance (LSPR) mode. The structure in Figure 3(c) consists of a cross and a bar [82]. Such a hybrid using an "X" and "I" shaped particles can generate Fano resonance caused by two relevant modes which are known as bonding mode (superradiant mode) and antibonding mode (subradiant mode). This nanoresonator can be applied in sensing applications since coherent coupling of bright and dark plasmon modes in this hybrid system is expected to produce Fano interference with high quality factors. Using nanoporous anodic alumina oxide (AAO) as templates, bimetallic (gold core with palladium shell) nanorod metamaterials with high aspect ratios [83] were fabricated using a self-organization technique (Figure 3(d)), which can find important applications in hydrogen gas sensing.

The key factor for strengthening the optical signals is the electric field enhancement assisted by plasmonic nanostructures. This has been extensively verified both theoretically and experimentally. As illustrated in Figure 4, near field increment generated by plasmonic nanostructures is obvious. 


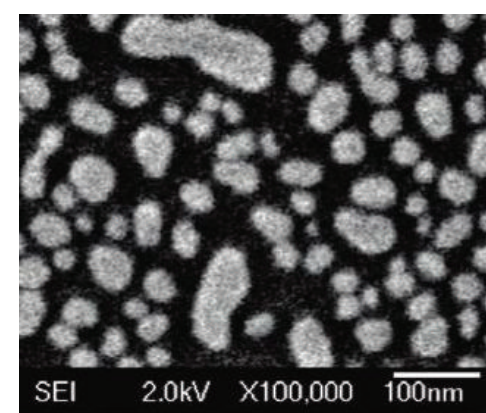

(a)

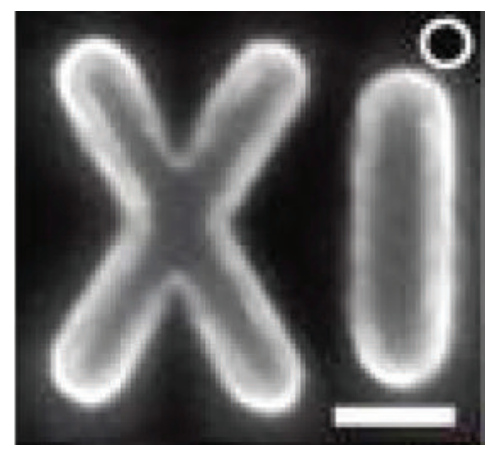

(c)

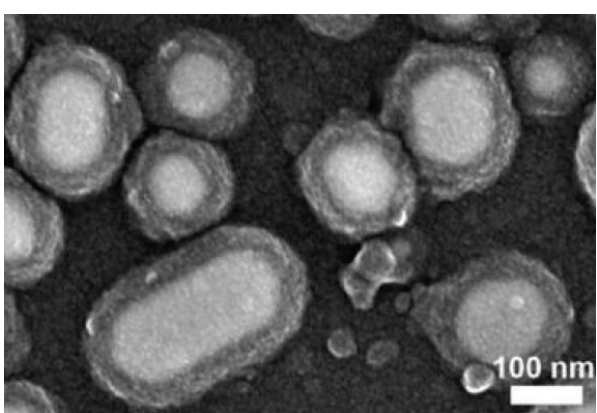

(b)

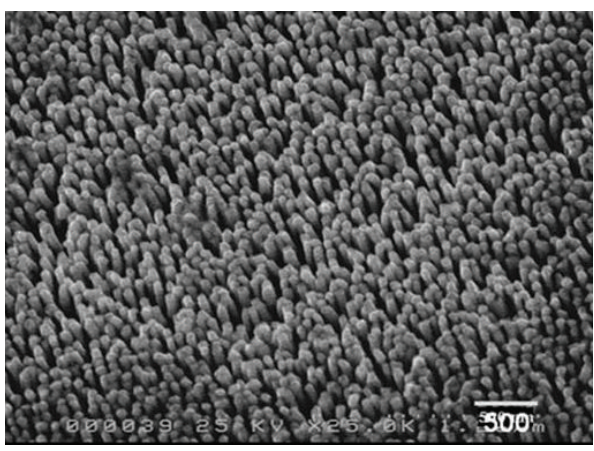

(d)

FIGURE 3: (a) and (b) SEM image of gold nanoislands fabricated by evaporation and annealing. (c) SEM image of a hybrid structure of X and I shaped particles. (d) SEM image of nanorods with high aspect ratios. Scale bar in (c), $100 \mathrm{~nm}$. (a), (b), (c), (d) are adapted from [80-83], respectively. (a): Copyright 2007, American Chemical Society. (b): Copyright 2011, American Chemical Society. (c): Copyright 2011, American Chemical Society. (d): Copyright 2014, Wiley.

By using different designs (e.g., nanocube particles and nanohole cavities) significantly increased near field intensity can be achieved. Enhanced electromagnetic fields are verified using finite difference time domain (FDTD) calculations. In Figures 4(a) and 4(b), one can see that different field intensity distributions are observed for different resonance modes (peaks) in scattering spectrum when a cubic silver particle is in contact with a dielectric substrate [84]. The silver cube with $90 \mathrm{~nm}$ length of side is supported by a glass substrate.

Since the dependence of field distribution can be either on the top of the particle (Figure 4(a)) or on the substrate (Figure 4(b)), one can apply this dependency to enhance the detecting signals for different kinds of analytes. The field distribution in Figure 4(c) demonstrates the enhancement effect using classic nanohole structures [85]. Exosomes supported by the nanohole plasmonic structures are thus sensed with larger signals. Since the typical size of exosomes is from $50 \mathrm{~nm}$ to $100 \mathrm{~nm}$ in diameter (the diameter and period of the hole array are $200 \mathrm{~nm}$ and $450 \mathrm{~nm}$, resp.), increased electromagnetic fields are confined in this range. Still using the classic nanohole arrays $(200 \mathrm{~nm}$ diameter and $600 \mathrm{~nm}$ periodicity), Cetin et al. demonstrated a plasmonic on-chip sensing platform [86] by covering a thin protein layer on the sample surface.

Quantitative detection of biomolecules with a wide range of concentrations (from $3.9 \mu \mathrm{g} / \mathrm{mL}$ to $1000 \mu \mathrm{g} / \mathrm{mL}$ ) is thus realized as shown in Figure 5(a). Spectral response (normalized transmission) reveals that the resonance peak redshifts with increasing concentrations. Liu and coworkers proposed a perfect absorber [87] using a thin layer of plasmonic nanodisks and further demonstrated its sensing application. Blueshift of resonance dips in the reflectance spectrum as a function of frequency was realized, as shown in Figure 5(b). More importantly, multispectral sensing can also be achieved by using proper plasmonic designs. A hexagonal crossshaped nanocavity array [88] was proposed and theoretically demonstrated to achieve narrowband near-unity absorption for sensing applications, as shown in Figure 5(c). We can see that the shift of resonance peaks in absorption spectrum is obvious for very small refractive index variation (1.000 to 1.040 with only 0.005 increments) from Figure 5 (c), which is potentially useful for detecting flammable gases and even poisonous materials [88]. Sensitivities of 448, 504, 538, and $564 \mathrm{~nm} / \mathrm{RIU}$ (from left to right) for the four resonance peaks are obtained, respectively.

Moreover, much higher sensitivity and figure of merit (near to the theoretical limit) have also been experimentally demonstrated using the nanomushrooms shown in Figure 2(d). As shown in Figure 6(a), the resonance dips in reflectance show distinct redshift with larger refractive indices [79]. For clarity, dips are normalized and replotted in Figure 6(b). $1015 \mathrm{~nm} / \mathrm{RIU}$ and 80-108 figures of merit are determined. 

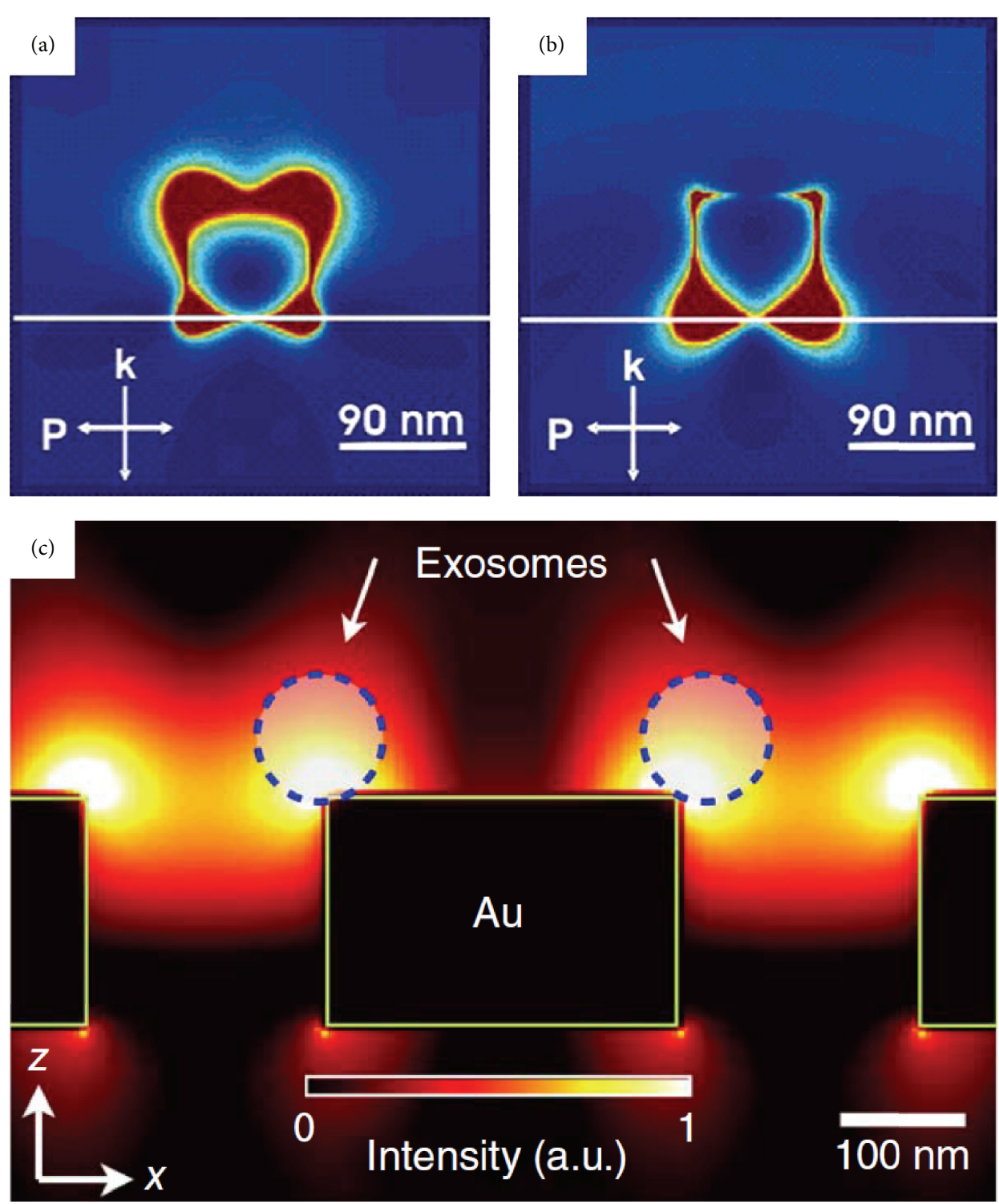

Figure 4: (a) and (b) The field intensities at different resonance peaks of a single silver nanocube in contact with a glass substrate. (c) The near field distribution showing enhanced electromagnetic fields tightly confined near a periodic nanohole surface. One can see that the field distribution overlaps with the size of exosomes captured onto the sensing surface. (a)-(b) and (c) are adapted from [84, 85], respectively. (a)-(b): Copyright 2005, American Chemical Society. (c): Copyright 2014, Nature Publishing Group.

\section{Conclusions and Outlook}

In summary, we have reviewed the typical sensing platforms using plasmonic designs with various nanostructures. By covering analytes on a sample surface, functional sensors with high performance (refractive index sensitivity and figure of merit) can be readily obtained since the electromagnetic field is dramatically enhanced at plasmon resonances. Different geometries (both well-aligned arrays and patterns with arbitrary shapes) can be utilized to actualize the enhanced sensing effect. Ultrahigh sensitivities enable various applications in a wide range of research domains. Since higher sensitivity and faster respond speed are always desired, future plasmonic sensors need ultrasensitive and ultrahigh speed performance. Moreover, sensing devices with high throughput and scalable detection are essential by combining plasmonics with microfluidics.

Graphene-related sensors [89-91] are emerging devices which have great potential and important applications. A graphene- $\mathrm{MoS}_{2}$ hybrid sensor [89] and an incident-angle tunable graphene-plasmonic sensor [90] are demonstrated most recently. The proposed graphene- $\mathrm{MoS}_{2}$ hybrid sensor has a huge phase-sensitivity enhancement compared with the SPR sensing scheme with only graphene coating since $\mathrm{MoS}_{2}$ 


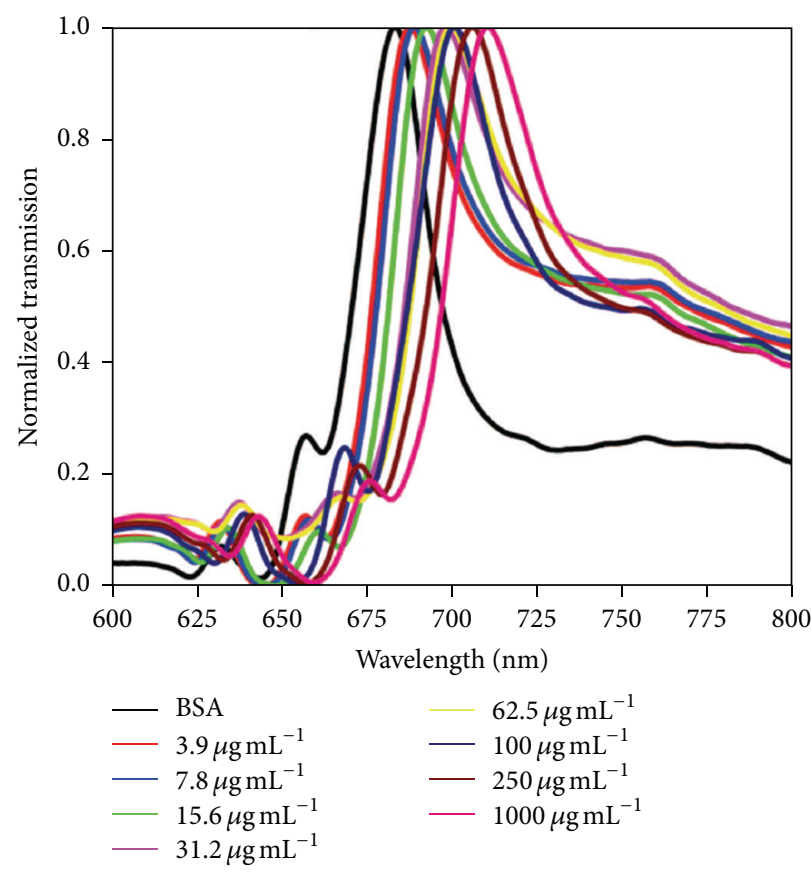

(a)

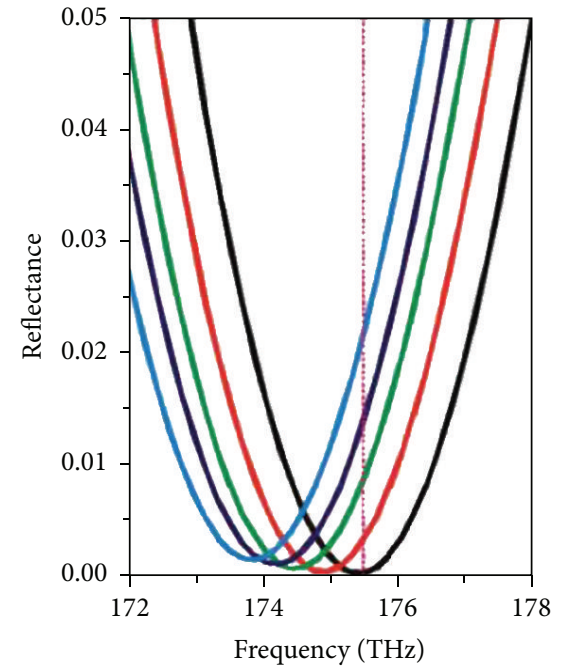

Water $(n=1.312)$

- Glucose solution ( $n=1.322)$

_ Glucose solution $(n=1.332)$

- Glucose solution $(n=1.342)$

_ Glucose solution $(n=1.352)$

(b)

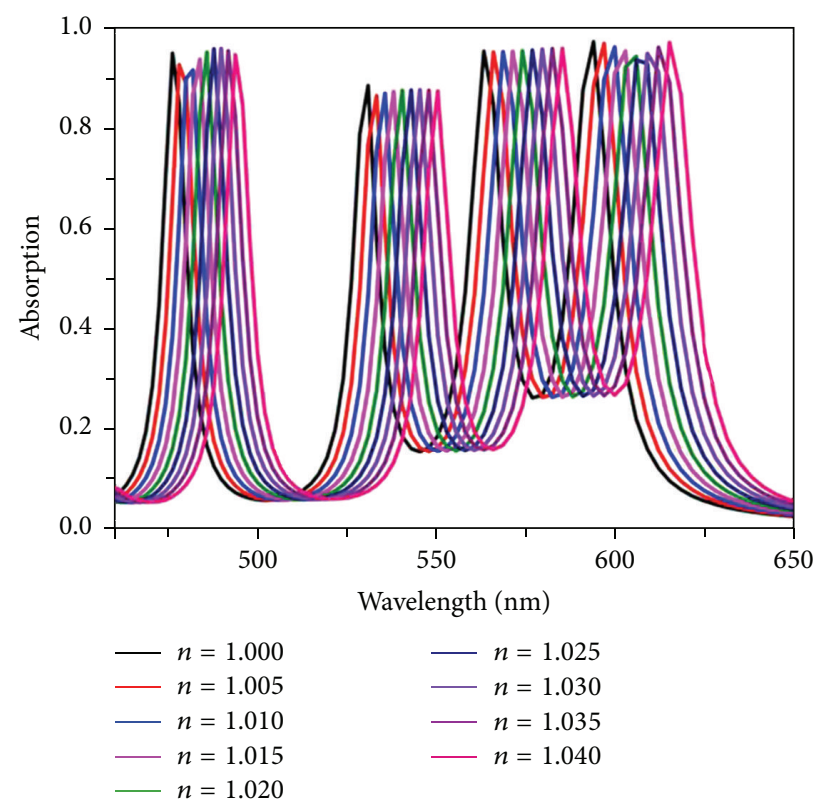

(c)

Figure 5: (a) Spectral response of the plasmonic sensors functionalized with different protein IgG concentrations ranging from $3.9 \mu \mathrm{g} / \mathrm{mL}$ to $1000 \mu \mathrm{g} / \mathrm{mL}$. (b) Reflectance spectra of an absorber sensor designed for water as reference medium. (c) Absorption spectra of the plasmonic nanostructure under a low refractive index environment. (a), (b), (c) are adapted from [86-88], respectively. (a): Copyright 2014, Nature Publishing Group. (b): Copyright 2010, American Chemical Society. (c): Copyright 2014, American Institute of Physics. 


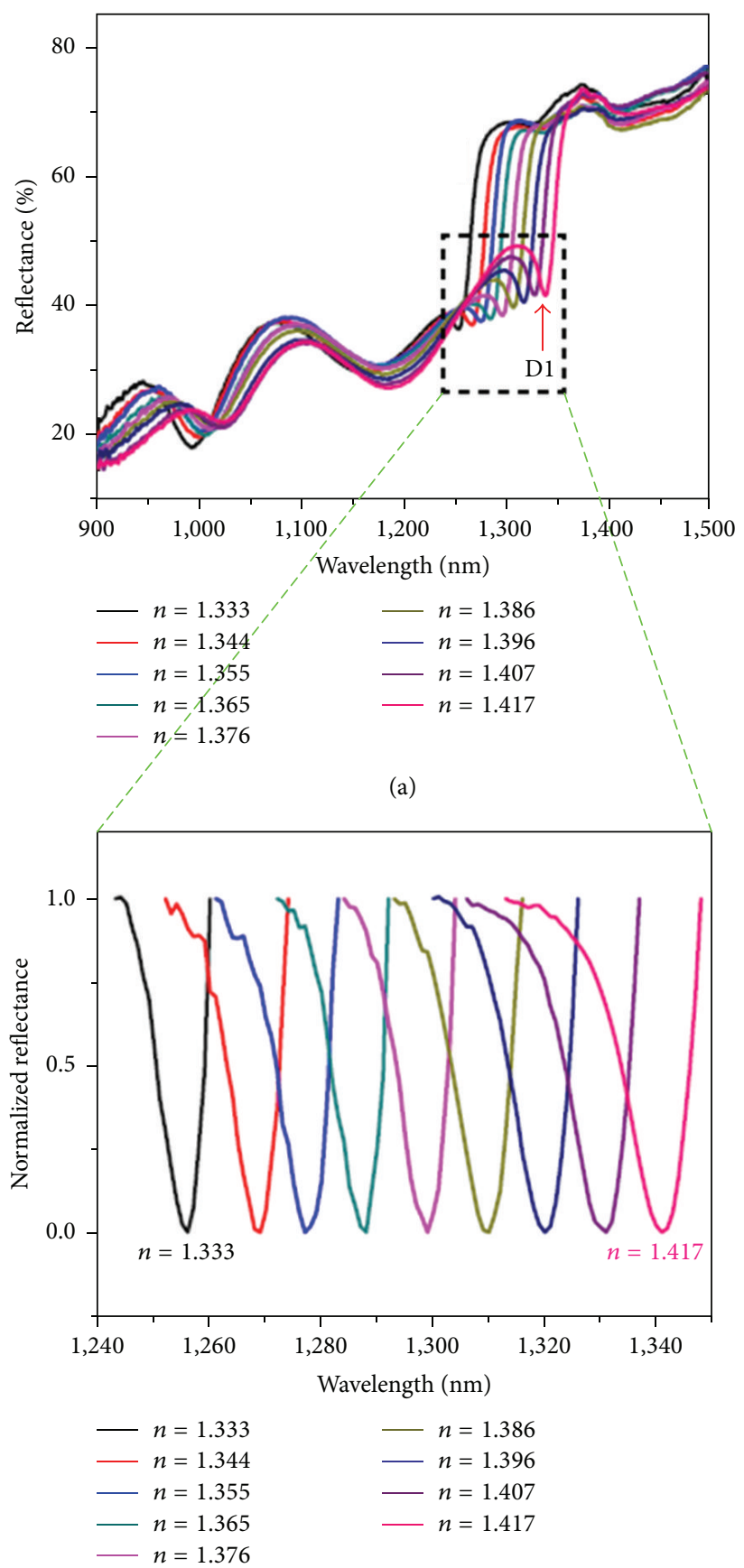

(b)

FIGURE 6: (a) Reflectance spectra of the gold mushroom array immersed in glycerine-water mixture solutions with varying compositions at the incidence angle of $33.3^{\circ}$. (b) Normalized reflectance for D1 in the spectral region indicated with the dashed box. (a) and (b) are adapted from [79]. (a)-(b): Copyright 2013, Nature Publishing Group.

has high absorption efficiency. For the incident-angle tunable graphene-plasmonic sensor, a TM-polarized light is needed to illuminate the structure beyond the critical angle with the help of a prism. Graphene has unsubstituted advantages such as high electronic mobility, large specific surface area, and preponderance of exposed edge planes to greatly increase charge storage and universal optical conductivity from visible to infrared frequencies and it has found tremendous application and great potential in composing bio/chemosensors.

\section{Conflict of Interests}

The authors declare that there is no conflict of interests regarding the publication of this paper. 


\section{Acknowledgments}

This work was partially supported by the National Natural Science Foundation of China (Grant no. 61405031). Leong and Liu acknowledge the Joint Council Office (JCO) of the Agency for Science, Technology and Research (A STAR) under Grant no. 12302FG012. Lv also acknowledges Natural Science Foundation of Hebei Province (Grant no. F2014501127), Science and Technology Research Funds for Higher Education of Hebei Province (Grant no. ZD2015209), and Fundamental Research Funds for the Central Universities (Grant no. N142304006).

\section{References}

[1] T. W. Ebbesen, H. J. Lezec, H. F. Ghaemi, T. Thio, and P. A. Wolff, "Extraordinary optical transmission through sub-wavelenght hole arrays," Nature, vol. 391, no. 6668, pp. 667-669, 1998.

[2] W. L. Barnes, A. Dereux, and T. W. Ebbesen, "Surface plasmon subwavelength optics," Nature, vol. 424, no. 6950, pp. 824-830, 2003.

[3] C. Genet and T. W. Ebbesen, "Light in tiny holes," Nature, vol. 445, no. 7123, pp. 39-46, 2007.

[4] H. J. Lezec, A. Degiron, E. Devaux et al., "Beaming light from a subwavelength aperture," Science, vol. 297, no. 5582, pp. 820$822,2002$.

[5] A. Rankin and S. McGarry, "A flexible pressure sensitive colour changing device using plasmonic nanoparticles," Nanotechnology, vol. 26, no. 7, Article ID 075502, 2015.

[6] M. Csete, G. Szekeres, A. Szenes, A. Szalai, and G. Szabó, "Plasmonic structure integrated single-photon detector configurations to improve absorptance and polarization contrast," Sensors, vol. 15, no. 2, pp. 3513-3539, 2015.

[7] T. Barman and A. R. Pal, "Plasmonic photosensitization of polyaniline prepared by a novel process for high-performance flexible photodetector," ACS Applied Materials and Interfaces, vol. 7, no. 4, pp. 2166-2170, 2015.

[8] N. Kholmicheva, P. Moroz, U. Rijal et al., "Plasmonic nanocrystal solar cells utilizing strongly confined radiation," ACS Nano, vol. 8, no. 12, pp. 12549-12559, 2014.

[9] H. Tanoto, J. H. Teng, Q. Y. Wu et al., "Nano-antenna in a photoconductive photomixer for highly efficient continuous wave terahertz emission," Scientific Reports, vol. 3, article 2824, 2013.

[10] Y. Li, H. Zhang, T. Mei, N. Zhu, D. H. Zhang, and J. Teng, "Effect of dielectric cladding on active plasmonic device based on InGaAsP multiple quantum wells," Optics Express, vol. 22, no. 21, pp. 25599-25607, 2014.

[11] Y. Xu, X. Wang, H. Deng, and K. Guo, "Tunable all-optical plasmonic rectifier in nanoscale metal-insulator-metal waveguides," Optics Letters, vol. 39, no. 20, pp. 5846-5849, 2014.

[12] X. Jiang, Q. Gu, F. Wang, J. Lv, Z. Ma, and G. Si, "Fabrication of coaxial plasmonic crystals by focused ion beam milling and electron-beam lithography," Materials Letters, vol. 100, pp. 192194, 2013.

[13] W. Wei, X. Zhang, and X. Ren, "Asymmetric hybrid plasmonic waveguides with centimeter-scale propagation length under subwavelength confinement for photonic components," Nanoscale Research Letters, vol. 9, article 599, 2014.

[14] Z. Zhang and J. Wang, "Long-range hybrid wedge plasmonic waveguide," Scientific Reports, vol. 4, article 6870, 2014.
[15] Y. Gao, G. Ren, B. Zhu, J. Wang, and S. Jian, "Single-mode graphene-coated nanowire plasmonic waveguide," Optics Letters, vol. 39, no. 20, pp. 5909-5912, 2014.

[16] M.-M. Jiang, H.-Y. Chen, C.-X. Shan, and D.-Z. Shen, “Tunability of hybridized plasmonic waveguide mediated by surface plasmon polaritons," Physical Chemistry Chemical Physics, vol. 16, no. 30, pp. 16233-16240, 2014.

[17] B. You, C.-C. Peng, J.-S. Jhang et al., "Terahertz plasmonic waveguide based on metal rod arrays for nanofilm sensing," Optics Express, vol. 22, no. 9, pp. 11340-11350, 2014.

[18] Z. Han, I. P. Radko, N. Mazurski et al., "On-chip detection of radiation guided by dielectric-loaded plasmonic waveguides," Nano Letters, vol. 15, no. 1, pp. 476-480, 2015.

[19] V. E. Babicheva, S. V. Zhukovsky, and A. V. Lavrinenko, "Bismuth ferrite as low-loss switchable material for plasmonic waveguide modulator," Optics Express, vol. 22, no. 23, pp. 28890-28897, 2014.

[20] H. E. Arabi, H.-E. Joe, T. Nazari, B.-K. Min, and K. Oh, "A high throughput supra-wavelength plasmonic bull's eye photon sorter spatially and spectrally multiplexed on silica optical fiber facet," Optics Express, vol. 21, no. 23, pp. 28083-28094, 2013.

[21] J. Le Perchec, Y. Desieres, N. Rochat, and R. Espiau de Lamaestre, "Subwavelength optical absorber with an integrated photon sorter," Applied Physics Letters, vol. 100, no. 11, Article ID 113305, 2012.

[22] E. Laux, C. Genet, T. Skauli, and T. W. Ebbesen, "Plasmonic photon sorters for spectral and polarimetric imaging," Nature Photonics, vol. 2, no. 3, pp. 161-164, 2008.

[23] A. De Luca, N. Depalo, E. Fanizza et al., "Plasmon mediated super-absorber flexible nanocomposites for metamaterials," Nanoscale, vol. 5, no. 13, pp. 6097-6105, 2013.

[24] X. Xiong, S.-C. Jiang, Y.-H. Hu, R.-W. Peng, and M. Wang, "Structured metal film as a perfect absorber," Advanced Materials, vol. 25, no. 29, pp. 3994-4000, 2013.

[25] W. Li, U. Guler, N. Kinsey et al., "Refractory plasmonics with titanium nitride: broadband metamaterial absorber," Advanced Materials, vol. 26, no. 47, pp. 7959-7965, 2014.

[26] G. Si, Y. Zhao, H. Liu et al., "Annular aperture array based color filter," Applied Physics Letters, vol. 99, no. 3, Article ID 033105, 2011.

[27] Y. J. Liu, G. Y. Si, E. S. P. Leong, N. Xiang, A. J. Danner, and J. H. Teng, "Light-driven plasmonic color filters by overlaying photoresponsive liquid crystals on gold annular aperture arrays," Advanced Materials, vol. 24, no. 23, pp. OP131-OP135, 2012.

[28] Y. J. Liu, G. Y. Si, E. S. P. Leong et al., "Optically tunable plasmonic color filters," Applied Physics A, vol. 107, no. 1, pp. 49-54, 2012.

[29] T. Xue, X. Cui, J. Chen et al., "A switch of the oxidation state of graphene oxide on a surface plasmon resonance chip," ACS Applied Materials and Interfaces, vol. 5, no. 6, pp. 2096-2103, 2013.

[30] S. Papaioannou, D. Kalavrouziotis, K. Vyrsokinos et al., "Active plasmonics in WDM traffic switching applications," Scientific Reports, vol. 2, article 652, 2012.

[31] V. Malyarchuk, F. Hua, N. H. Mack et al., "High performance plasmonic crystal sensor formed by soft nanoimprint lithography," Optics Express, vol. 13, no. 15, pp. 5669-5675, 2005.

[32] K.-S. Lee and M. A. El-Sayed, "Gold and silver nanoparticles in sensing and imaging: sensitivity of plasmon response to size, shape, and metal composition," The Journal of Physical Chemistry B, vol. 110, no. 39, pp. 19220-19225, 2006. 
[33] F. Goettmann, A. Moores, C. Boissière, P. Le Floch, and C. Sanchez, "A selective chemical sensor based on the plasmonic response of phosphinine-stabilized gold nanoparticles hosted on periodically organized mesoporous silica thin layers," Small, vol. 1, no. 6, pp. 636-639, 2005.

[34] S. M. Marinakos, S. Chen, and A. Chilkoti, "Plasmonic detection of a model analyte in serum by a gold nanorod sensor," Analytical Chemistry, vol. 79, no. 14, pp. 5278-5283, 2007.

[35] J. N. Anker, W. P. Hall, O. Lyandres, N. C. Shah, J. Zhao, and R. P. Van Duyne, "Biosensing with plasmonic nanosensors," Nature Materials, vol. 7, no. 6, pp. 442-453, 2008.

[36] A. Hassani and M. Skorobogatiy, "Surface plasmon resonancelike integrated sensor at terahertz frequencies for gaseous analytes," Optics Express, vol. 16, no. 25, pp. 20206-20214, 2008.

[37] G. J. Nusz, A. C. Curry, S. M. Marinakos, A. Wax, and A. Chilkoti, "Rational selection of gold nanorod geometry for label-free plasmonic biosensors," ACS Nano, vol. 3, no. 4, pp. 795-806, 2009.

[38] A. B. Dahlin, S. Chen, M. P. Jonsson, L. Gunnarsson, M. Käll, and F. Höök, "High-resolution microspectroscopy of plasmonic nanostructures for miniaturized biosensing," Analytical Chemistry, vol. 81, no. 16, pp. 6572-6580, 2009.

[39] M. A. Otte, B. Sepúlveda, W. Ni, J. P. Juste, L. M. Liz-Marzán, and L. M. Lechuga, "Identification of the optimal spectral region for plasmonic and nanoplasmonic sensing," ACS Nano, vol. 4, no. 1, pp. 349-357, 2010.

[40] Y.-T. Chang, Y.-C. Lai, C.-T. Li, C.-K. Chen, and T.-J. Yen, "A multi-functional plasmonic biosensor," Optics Express, vol. 18, no. 9, pp. 9561-9569, 2010.

[41] S. Ahn, S. Y. Jung, J. P. Lee, H. K. Kim, and S. J. Lee, "Gold nanoparticle flow sensors designed for dynamic X-ray imaging in biofluids," ACS Nano, vol. 4, no. 7, pp. 3753-3762, 2010.

[42] L. E. Kreno, J. T. Hupp, and R. P. Van Duyne, "Metal-organic framework thin film for enhanced localized surface plasmon resonance gas sensing," Analytical Chemistry, vol. 82, no. 19, pp. 8042-8046, 2010.

[43] C. Escobedo, A. G. Brolo, R. Gordon, and D. Sinton, "Flowthrough vs flow-over: analysis of transport and binding in nanohole array plasmonic biosensors," Analytical Chemistry, vol. 82, no. 24, pp. 10015-10020, 2010.

[44] M.-S. Kwon, "Disposable and compact integrated plasmonic sensor using a long-period grating," Optics Letters, vol. 35, no. 22, pp. 3835-3837, 2010.

[45] B. Khademhosseinieh, G. Biener, I. Sencan, T.-W. Su, A. F. Coskun, and A. Ozcan, "Lensfree sensing on a microfluidic chip using plasmonic nanoapertures," Applied Physics Letters, vol. 97, no. 22, Article ID 221107, 2010.

[46] A. François, J. Boehm, S. Y. Oh, T. Kok, and T. M. Monro, "Collection mode surface plasmon fibre sensors: a new biosensing platform," Biosensors and Bioelectronics, vol. 26, no. 7, pp. 31543159, 2011.

[47] J. D. Caldwell, O. Glembocki, F. J. Bezares et al., "Plasmonic nanopillar arrays for large-area, high-enhancement surfaceenhanced Raman scattering sensors," ACS Nano, vol. 5, no. 5, pp. 4046-4055, 2011.

[48] C.-Y. Tsai, S.-P. Lu, J.-W. Lin, and P.-T. Lee, "High sensitivity plasmonic index sensor using slablike gold nanoring arrays," Applied Physics Letters, vol. 98, no. 15, Article ID 153108, 2011.

[49] L. Guyot, A.-P. Blanchard-Dionne, S. Patskovsky, and M. Meunier, "Integrated silicon-based nanoplasmonic sensor," Optics Express, vol. 19, no. 10, pp. 9962-9967, 2011.
[50] B. Jose, C. T. Mallon, R. J. Forster, and T. E. Keyes, "Regio-selective decoration of nanocavity metal arrays: contributions from localized and delocalized plasmons to surface enhanced Raman spectroscopy," Physical Chemistry Chemical Physics, vol. 13, no. 32, pp. 14705-14714, 2011.

[51] Y. Zhang, C. Zhou, L. Xia, X. Yu, and D. Liu, "Wagon wheel fiber based multichannel plasmonic sensor," Optics Express, vol. 19, no. 23, pp. 22863-22873, 2011.

[52] M. Maisonneuve, O. D. A. Kelly, A.-P. Blanchard-Dionne, S. Patskovsky, and M. Meunier, "Phase sensitive sensor on plasmonic nanograting structures," Optics Express, vol. 19, no. 27, pp. 26318-26324, 2011.

[53] T. B. Andersen, Z. Han, and S. I. Bozhevolnyi, "Compact onchip temperature sensors based on dielectric-loaded plasmonic waveguide-ring resonators," Sensors, vol. 11, no. 2, pp. 19922000, 2011.

[54] P. Jia, H. Jiang, J. Sabarinathan, and J. Yang, "Plasmonic nanohole array sensors fabricated by template transfer with improved optical performance," Nanotechnology, vol. 24, no. 19, Article ID 195501, 2013.

[55] E. Kazuma and T. Tatsuma, "Localized surface plasmon resonance sensors based on wavelength-tunable spectral dips," Nanoscale, vol. 6, no. 4, pp. 2397-2405, 2014.

[56] K.-S. Lee, J. M. Son, D.-Y. Jeong, T. S. Lee, and W. M. Kim, “Resolution enhancement in surface plasmon resonance sensor based on waveguide coupled mode by combining a bimetallic approach," Sensors, vol. 10, no. 12, pp. 11390-11399, 2010.

[57] G. Si, Y. Zhao, E. S. P. Leong, and Y. J. Liu, "Liquid-crystalenabled active plasmonics: a review," Materials, vol. 7, no. 2, pp. 1296-1317, 2014.

[58] A. Dhawan, M. Canva, and T. Vo-Dinh, "Narrow groove plasmonic nano-gratings for surface plasmon resonance sensing," Optics Express, vol. 19, no. 2, pp. 787-813, 2011.

[59] M. A. van de Haar, R. Maas, H. Schokker, and A. Polman, "Experimental realization of a polarization-independent ultraviolet/visible coaxial plasmonic metamaterial," Nano Letters, vol. 14, no. 11, pp. 6356-6360, 2014.

[60] H. Kollmann, X. Piao, M. Esmann et al., "Toward plasmonics with nanometer precision: nonlinear optics of helium-ion milled gold nanoantennas," Nano Letters, vol. 14, no. 8, pp. 4778-4784, 2014.

[61] G. Si, Y. Zhao, J. Lv et al., "Direct and accurate patterning of plasmonic nanostructures with ultrasmall gaps," Nanoscale, vol. 5, no. 10, pp. 4309-4313, 2013.

[62] G. Han, D. Weber, F. Neubrech et al., "Infrared spectroscopic and electron microscopic characterization of gold nanogap structure fabricated by focused ion beam," Nanotechnology, vol. 22, no. 27, Article ID 275202, 2011.

[63] T. Zhang, S. Callard, C. Jamois, C. Chevalier, D. Feng, and A. Belarouci, "Plasmonic-photonic crystal coupled nanolaser," Nanotechnology, vol. 25, no. 31, Article ID 315201, 2014.

[64] G. Y. Si, E. S. P. Leong, W. Pan, C. C. Chum, and Y. J. Liu, "Plasmon-induced transparency in coupled triangle-rod arrays," Nanotechnology, vol. 26, no. 2, Article ID 025201, 2015.

[65] A. B. Taylor, P. Michaux, A. S. M. Mohsin, and J. W. M. Chon, "Electron-beam lithography of plasmonic nanorod arrays for multilayered optical storage," Optics Express, vol. 22, no. 11, pp. 13234-13243, 2014.

[66] R. Near, C. Tabor, J. Duan, R. Pachter, and M. El-Sayed, "Pronounced effects of anisotropy on plasmonic properties of nanorings fabricated by electron beam lithography," Nano Letters, vol. 12, no. 4, pp. 2158-2164, 2012. 
[67] G. Si, Y. Zhao, J. Lv et al., "Reflective plasmonic color filters based on lithographically patterned silver nanorod arrays," Nanoscale, vol. 5, no. 14, pp. 6243-6248, 2013.

[68] E. S. P. Leong, S. Wu, N. Zhang et al., "Optical properties of ultrafine line and space polymeric nanogratings coated with metal and metal-dielectric-metal thin films," Nanotechnology, vol. 25, no. 5, Article ID 055203, 2014.

[69] C. L. C. Smith, B. Desiatov, I. Goykmann, I. Fernandez-Cuesta, U. Levy, and A. Kristensen, "Plasmonic V-groove waveguides with Bragg grating filters via nanoimprint lithography," Optics Express, vol. 20, no. 5, pp. 5696-5706, 2012.

[70] C.-C. Yu, K.-H. Ho, H.-L. Chen, S.-Y. Chuang, S.-C. Tseng, and W.-F. Su, "Using the nanoimprint-in-metal method to prepare corrugated metal structures for plasmonic biosensors through both surface plasmon resonance and index-matching effects," Biosensors and Bioelectronics, vol. 33, no. 1, pp. 267-273, 2012.

[71] H.-W. Yoo, J.-M. Jung, S.-K. Lee, and H.-T. Jung, "The fabrication of highly ordered silver nanodot patterns by platinum assisted nanoimprint lithography," Nanotechnology, vol. 22, no. 9, Article ID 095304, 2011.

[72] G. Si, Y. Zhao, E. S. P. Leong, J. Lv, and Y. J. Liu, "Incidentangle dependent color tuning from a single plasmonic chip," Nanotechnology, vol. 25, no. 45, Article ID 455203, 2014.

[73] G. Si, X. Jiang, J. Lv, Q. Gu, and F. Wang, "Fabrication and characterization of well-aligned plasmonic nanopillars with ultrasmall separations," Nanoscale Research Letters, vol. 9, article 299, 2014.

[74] H.-J. Ahn, P. Thiyagarajan, L. Jia et al., "An optimal substrate design for SERS: dual-scale diamond-shaped gold nano-structures fabricated via interference lithography," Nanoscale, vol. 5, no. 5, pp. 1836-1842, 2013.

[75] M. Jin, H. van Wolferen, H. Wormeester, A. van den Berg, and E. T. Carlen, "Large-area nanogap plasmon resonator arrays for plasmonics applications," Nanoscale, vol. 4, no. 15, pp. 47124718, 2012.

[76] B. Zeng, Y. Gao, and F. J. Bartoli, "Rapid and highly sensitive detection using Fano resonances in ultrathin plasmonic nanogratings," Applied Physics Letters, vol. 105, Article ID 161106, 2014.

[77] G. Si, Q. Wang, J. Lv, L. Miao, F. Wang, and S. Peng, "Interference lithography patterned large area plasmonic nanodisks for infrared detection," Materials Letters, vol. 128, pp. 373-375, 2014.

[78] Z. L. Cao, S. L. Wong, S. Y. Wu, H. P. Ho, and H. C. Ong, "High performing phase-based surface plasmon resonance sensing from metallic nanohole arrays," Applied Physics Letters, vol. 104, no. 17, Article ID 171116, 2014.

[79] Y. Shen, J. Zhou, T. Liu et al., "Plasmonic gold mushroom arrays with refractive index sensing figures of merit approaching the theoretical limit," Nature Communications, vol. 4, article 2381, 2013.

[80] I. Ruach-Nir, T. A. Bendikov, I. Doron-Mor, Z. Barkay, A. Vaskevich, and I. Rubinstein, "Silica-stabilized gold island films for transmission localized surface plasmon sensing," Journal of the American Chemical Society, vol. 129, no. 1, pp. 84-92, 2007.

[81] O. Kedem, A. B. Tesler, A. Vaskevich, and I. Rubinstein, "Sensitivity and optimization of localized surface plasmon resonance transducers," ACS Nano, vol. 5, no. 2, pp. 748-760, 2011.

[82] N. Verellen, P. Van Dorpe, C. Huang et al., "Plasmon line shaping using nanocrosses for high sensitivity localized surface plasmon resonance sensing," Nano Letters, vol. 11, no. 2, pp. 391397, 2011.
[83] M. E. Nasir, W. Dickson, G. A. Wurtz, W. P. Wardley, and A. V. Zayats, "Hydrogen detected by the naked eye: optical hydrogen gas sensors based on core/shell plasmonic nanorod metamaterials," Advanced Materials, vol. 26, no. 21, pp. 35323537, 2014.

[84] L. J. Sherry, S.-H. Chang, G. C. Schatz, R. P. Van Duyne, B. J. Wiley, and Y. Xia, "Localized surface plasmon resonance spectroscopy of single silver nanocubes," Nano Letters, vol. 5, no. 10, pp. 2034-2038, 2005.

[85] H. Im, H. Shao, Y. I. Park et al., "Label-free detection and molecular profiling of exosomes with a nano-plasmonic sensor," Nature Biotechnology, vol. 32, no. 5, pp. 490-495, 2014.

[86] A. E. Cetin, A. F. Coskun, B. C. Galarreta et al., "Handheld highthroughput plasmonic biosensor using computational on-chip imaging," Light: Science and Applications, vol. 3, article e122, Article ID 20143, 2014.

[87] N. Liu, M. Mesch, T. Weiss, M. Hentschel, and H. Giessen, "Infrared perfect absorber and its application as plasmonic sensor," Nano Letters, vol. 10, no. 7, pp. 2342-2348, 2010.

[88] Z. Liu, H. Shao, G. Liu et al., " $\lambda^{3} / 20000$ plasmonic nanocavities with multispectral ultra-narrowband absorption for highquality sensing," Applied Physics Letters, vol. 104, Article ID 081116, 2014.

[89] S. Zeng, S. Hu, J. Xia et al., "Graphene- $\mathrm{MoS}_{2}$ hybrid nanostructures enhanced surface plasmon resonance biosensors," Sensors and Actuators B: Chemical, vol. 207, pp. 801-810, 2015.

[90] P. K. Maharana, R. Jha, and P. Padhy, "On the electric field enhancement and performance of SPR gas sensor based on graphene for visible and near infrared," Sensors and Actuators B: Chemical, vol. 207, pp. 117-122, 2015.

[91] K. V. Sreekanth, S. Zeng, K.-T. Yong, and T. Yu, "Sensitivity enhanced biosensor using graphene-based one-dimensional photonic crystal," Sensors and Actuators, B: Chemical, vol. 182, pp. 424-428, 2013. 

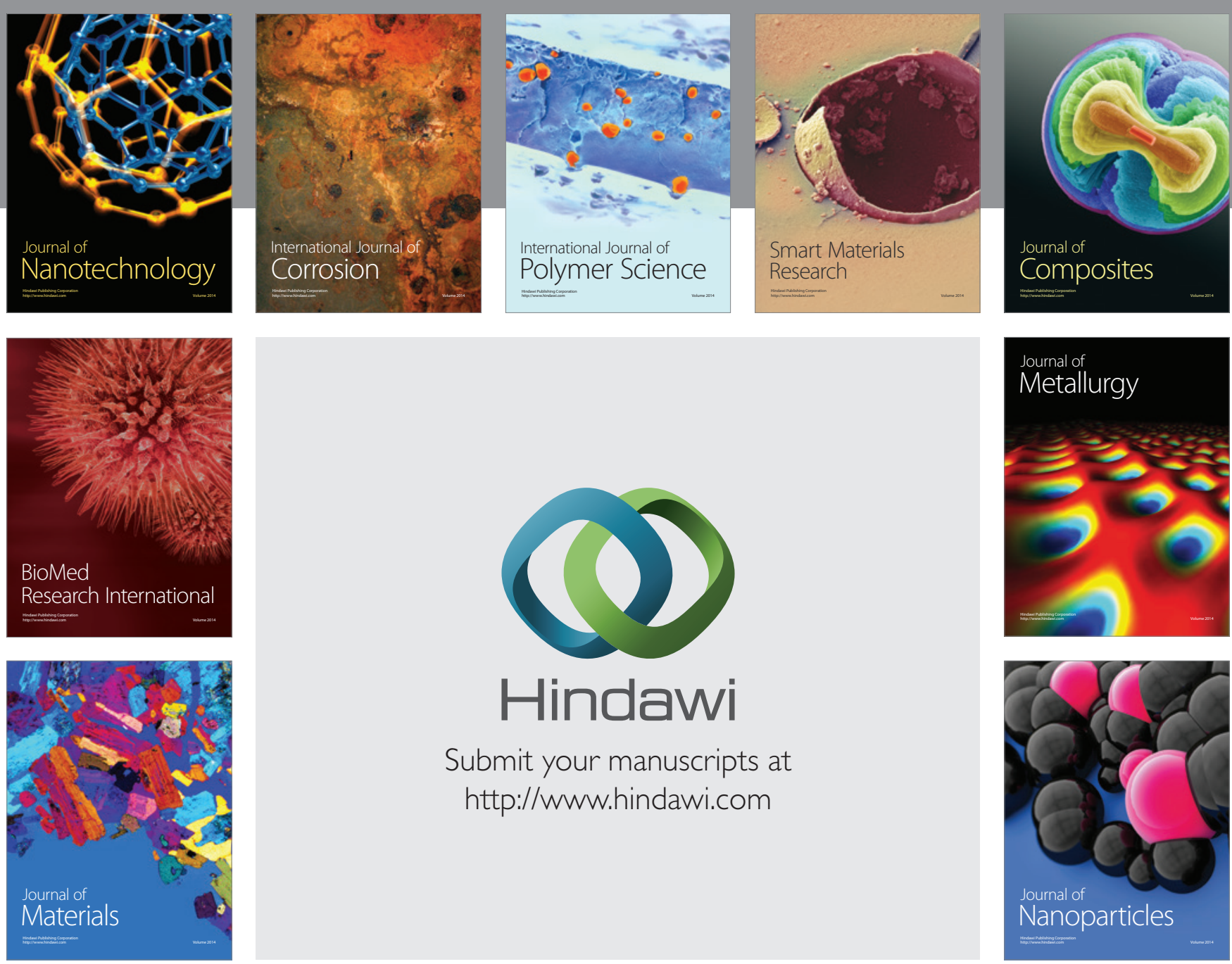

Submit your manuscripts at http://www.hindawi.com
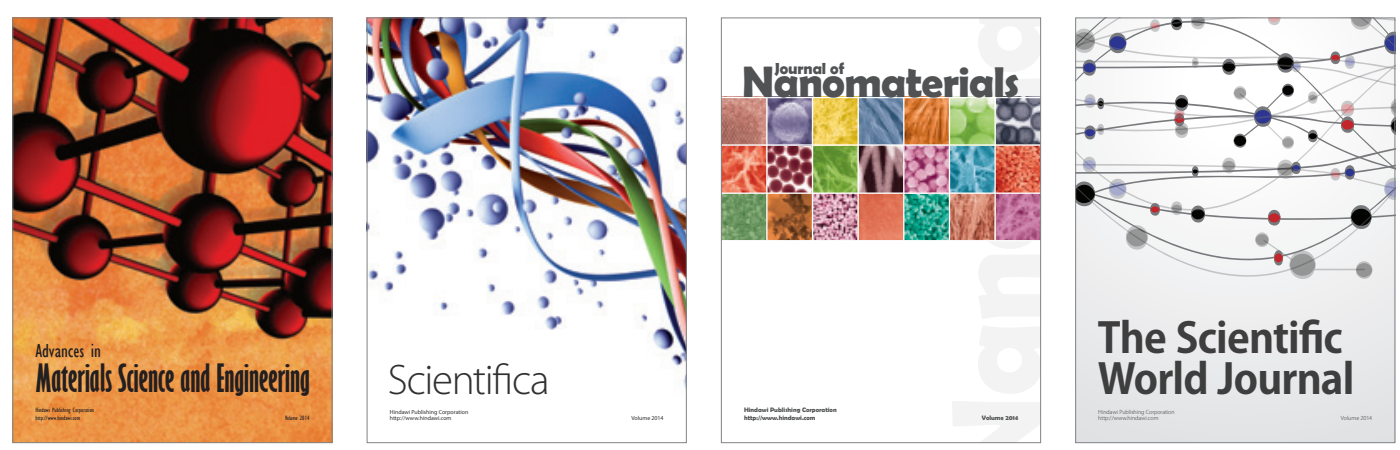

\section{The Scientific World Journal}
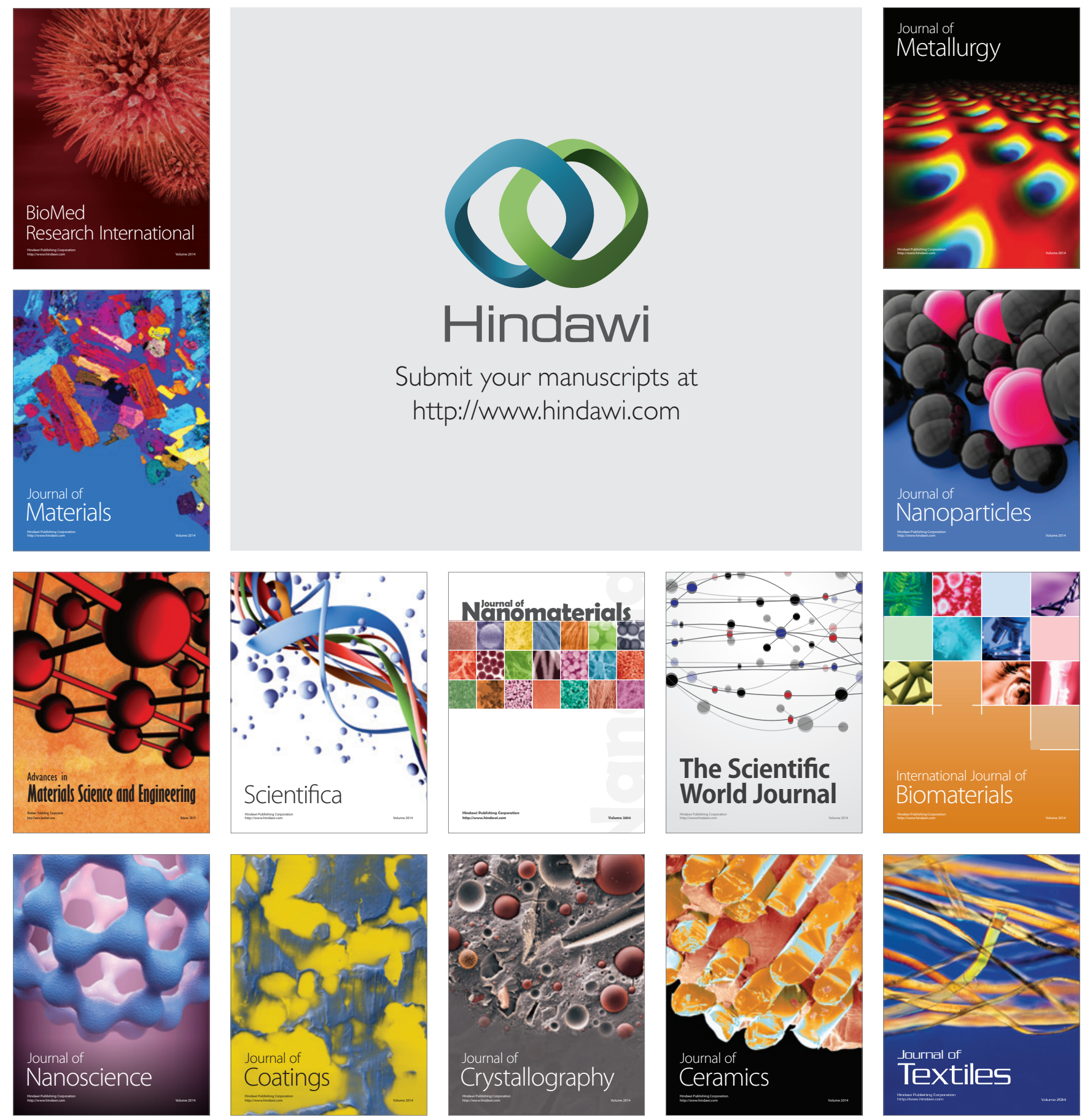